\title{
A long-expected party
}

\author{
The awarding of this year's Nobel Prize in Chemistry for the development of lithium-ion batteries was long overdue \\ for a technology that is already providing a vital component of the energy economy.
}

O nly the phenomenal vitality of John Goodenough has saved the Nobel committee from embarrassment. Calls for his pioneering work on lithium batteries to be rewarded have been a routine aspect of Nobel predictions for many years, and finally the time has arrived. Now aged 97, Goodenough is the oldest person ever to win one of the prizes. He shared the chemistry award with Stanley Whittingham of Binghamton University, State University of New York, and Akira Yoshino of the Asahi Kasei Corporation in Tokyo.

Few decisions better exemplify Alfred Nobel's desire to reward "those who ... shall have conferred the greatest benefit to humankind." And few better illustrate how research that might at first have seemed somewhat narrow in scope can find global significance. When Whittingham began to develop materials for lithium-battery cathodes at Exxon research in the 1970s, this seemed like a promising avenue to a useful new category of rechargeable batteries - a technology given some urgency at that time because of the oil crisis. Few then appreciated that an energy economy less dependent on fossil fuels would be needed not as insulation against fluctuating oil prices but to avoid the much graver threat of instability in the planetary system.

Batteries are of course a store rather than a primary source of energy. But rechargeable devices with good cyclability and high energy density can not only capture and distribute renewable energy from intermittent sources such as wind and solar power, but also ease us away from a transportation infrastructure that depends on gasoline and other carbon-based fuels.

Whittingham's first devices used layered titanium disulfide as the lithium-ionintercalating cathode, and lithium metal as the anode ${ }^{1}$. His discovery of lithium-ion intercalation into sulfides was serendipitous, arising from work on potassium doping of tantalum sulfide to enhance its superconductivity (although alkali-metal intercalation compounds of sulfides had been studied earlier by Rouxel and co-workers ${ }^{2}$ ). Titanium replaced tantalum in the electrode to lower the cost ${ }^{3}$.

In 1980 Goodenough and co-workers, working at the University of Oxford, improved on the design in an early demonstration of the value of an informed

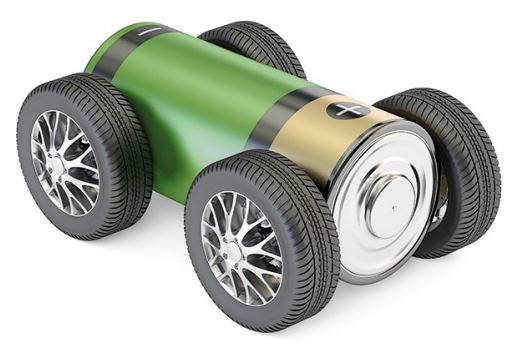

Credit: Olekcii Mach/Alamy Stock Photo

yet open-ended exploration of materials space. Recognizing that metal oxides would have a higher voltage than sulfides, he identified cobalt oxide as a lithium intercalator that was stable and could sustain cycling at relatively high rates.

Lithium metal is of course highly reactive, raising dangers about the stability and safety of the batteries. The first lithium batteries marketed in the 1980s for portable electronics were dogged by a tendency to burst into flames. Yoshino cracked the anode problem in 1985, showing that a disordered form of graphite (petroleum coke) could replace lithium metal while developing a high enough potential. In these 'rockingchair' cells the ions simply shuttle in and out of layered materials that absorb them with relatively little degradation ${ }^{5}$.

Only then did lithium batteries become a serious contender in the market for rechargeable cells, then dominated by lead-acid batteries for vehicles and nickel-cadmium cells for electronics, with nickel-metal hydride batteries vying for a place in both. Japanese investment in the technology was motivated by the nation's central role in the emergence of wireless consumer electronics: Sony boldly moved ahead to create the first commercial cells in 1991. Yet lithium-ion batteries were still not free from hazard, in part due to the tendency of lithium metal to 'plate' onto the carbon anode during recharging. Shortcircuiting caused a serious fire in one British manufacturing plant in 1995, the batteries reported as "exploding like missiles." Yet steady improvements in both safety and performance mean that lithium-ion batteries have become the standard power source for handheld consumer electronics, with a projected market of around US $\$ 2$ billion by 2024. They are also currently preferred for electric vehicles; the current driving range without recharge is around 300 miles, but that should increase in time.

Still it remains challenging to make lithium batteries with the capacity and power density needed for long-term applications such as heavy goods vehicles and the electrification of light aircraft, where energy stored per unit mass becomes crucial. So there is much interest in alternative battery technologies, including all-solid-state (see the Review by Masquelier and colleagues in this issue), lithium-sulfur and lithium-air batteries ${ }^{6}$. The latter can have a higher energy density than current lithium-ion batteries, but they revert to lithium metal anodes. Metal redeposition during charging is then difficult to manage, again potentially producing dendrite needles that cause short-circuits. Overcoming such drawbacks demands ingenious materials solutions, or possibly replacing lithium by more abundant metals such as sodium, magnesium and calcium ${ }^{7}$.

If there is a general lesson here, it is that technological innovation is highly nonlinear. The lithium battery first surfaced as a hedge against uncertainties in fuel prices, not as a 'green' technology. Its key market opened up only because other developments in electronics - enabling mobile phones and laptop computers - created a niche. The widespread adoption of electric vehicles using lithium-ion batteries, necessary to mitigate climate change, will depend both on state-imposed incentives (for example, to phase out gasoline vehicles) and investment in the necessary infrastructure. Research can't anticipate such contingencies which is why it pays to invest in creative researchers and keep options open for all types of battery technologies.

Published online: 20 November 2019 https://doi.org/10.1038/s41563-019-0554-6

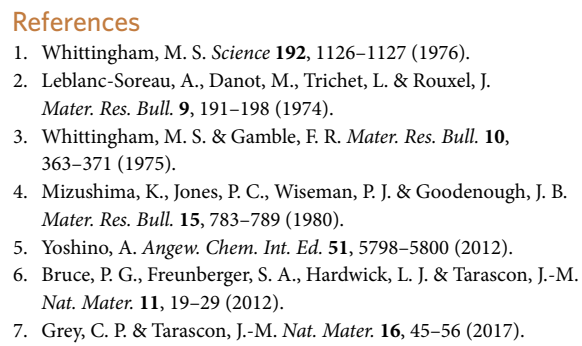

